\title{
Mobile Application for Emergency Navigation During Disaster Using Wireless Sensor Network
}

\author{
Rahul Krishnan, Kannan G., Mathibala G. \\ Department of Electronics and Communication Engineering, Rajalakshmi Institute of Technology, Chennai, India \\ Email address: \\ rahulkrish1990@gmail.com (R. Krishnan) \\ To cite this article: \\ Rahul Krishnan, Kannan G., Mathibala G. Mobile Application for Emergency Navigation During Disaster Using Wireless Sensor Network. \\ Advances in Wireless Communications and Networks. Vol. 4, No. 1, 2018, pp. 1-4. doi: 10.11648/j.awcn.20180401.11
}

Received: May 2, 2018; Accepted: May 30, 2018; Published: June 19, 2018

\begin{abstract}
This Wireless Sensor Network (WSN) is gathering sensor hubs. A huge number of sensor hubs associated with each frame sensor arrange. Sensor hubs ordinarily comprise of radio handset, microcontroller and it is fueled with battery. In this Mobile Environment, the clients are furnished with Personal Digital Assistants (PDA) or advanced cells that can converse with the Sensors effectively. At the point when crisis happens, the WSN gives essential data to clients, So that guided to move out of an unsafe zone through connection with sensors. Remote system sensor joined with a route calculation could encourage securely control individuals to a building exit while helping them evade perilous range. We propose a plain route calculation for crisis circumstance. Clog Adaptive and little extend crisis Navigation calculation with WSNs (CANS) use level set strategy to track the development of the exit and the limit of the perilous territory, so that individuals close-by the risky region accomplish a gentle blockage at the cost of a slight reroute, while individuals inaccessible from the threat evade superfluous alternate routes. Firstly, the route of people looks for a safe-basic way, other than parcel misfortune or vitality productivity which is the primary need as in bundle directing. Besides, human route expends a great deal additional time than customary parcel directing procedure, because of the constrained development speed of individuals. Also, which are basic for a quick clearing, as they primarily concentrate on finding the briefest/most secure way for every individual, while other problematic (yet sheltered) ways are left unused all through a large portion of the departure procedure.
\end{abstract}

Keywords: Wireless Sensor Network, Personal Digital Assistant, Emergency Navigation, Throughput

\section{Introduction}

In wireless sensor networks, numerous reasonable and little sensor-rich gadgets are sent to screen and control our condition [2]. Every gadget, called a sensor hub, is equipped for detecting, calculation, and correspondence. Sensor hubs shape a remote specially appointed system for correspondence. The restricted supply of force and different limitations, for example, fabricating expenses and constrained bundle sizes, confine the abilities of every sensor hub. For instance, a common sensor hub has short correspondence and detecting ranges, a restricted measure of memory, and constrained computational power. Notwithstanding, the bounteous number of spatially spread sensors will empower us to screen changes in our condition precisely regardless of the incorrectness of every sensor hub.

One of the Major utilizations of Wireless Sensor Network (WSNs) is the route benefit for crisis clearing. It is generally utilized as a part of different spaces, for example, military application, industrials and condition. Crisis responders require area and route bolster however couple of business research area frameworks are plan in view of them. The route application was connection amongst sensor and client. In this paper introduced on CANS is the primary WSN helped crisis route calculation accomplishing both gentle clog and little extend, where all operations are in-situ done by digital physical cooperation among individuals and sensor hubs.

Jars use level set technique to track the development of the exit and the limit of the perilous zone, so that individuals close- by the dangerous territory accomplish a gentle blockage at the cost of a slight reroute, while individuals inaccessible from the threat maintain a strategic distance from pointless alternate routes. Jars do not require area data, and CANS Algorithm contain level arrangement of techniques. The main strategy to Establishing the Potential guide, second technique to Building the Hazard level guide 
and last technique is planning a protected way for every client (Potential guide and Hazard level guide join Compound level guide). At the point when crisis happens existing framework just concentrate on finding the most secure way for every individual, except they are not considering the blockage amid the sensor trigger time. It is one of the real issues in this framework. The option way won't be appeared on a similar time while overlooking an indirect way briefly supplanting some portion of a course. The principle motivation behind this paper is to help individuals in getting away from an unsafe (risky region) area immediately when a crisis happens with ensured wellbeing, while maintaining a strategic distance from unreasonable blockages and superfluous temporary routes. The rest of this paper is organized as takes after. The audit of various works is portrayed in Section 2. In Section 3, proposed engineering and reasonable clarifications are portrayed. The conclusion is given in Sections 4 .

\section{Related Works}

Jiliang Wang et.al [7] proposed a usage of the sensor network infrastructure for the navigation of the users during danger. The successive objective of this idea is the addition of human safety and time factors. In this paper we bring up an idea to embed a road map system in a sensor network without location information which can provide the users with navigation routes and guaranteed safety. We also design an efficient road map updating mechanism to rebuild the roadmap when any changes occur. In this navigation system each user obtains their navigation route, this supports multiple users simultaneously. To examine the efficiency and scalability of this approach a large scale simulation is been conducted on various environments.

Chen Wang et.al [1] brings up an idea based on constructing a space filling curve (SFC) which linearizes the high genus 3 dimensional surfaces WSN, which yields a traversal path. SURF first constructs the iso- contour in discrete settings with the use of hop-count distance function, then the Reeb graph concept is been used to divide the network scheme. Finally, a novel serial traversal scheme is been conducted which enables the traversal within and between the regions. SURF is the first purely connectivity based solution for the linearization of the networks. It is advantageous as it is fully distributed and highly scalable, which also requires a constant storage. It has been demonstrated that extensive simulations on several networks works well on high genus 3D surface WSNs with the help of SURF.

Zheng Zhang et.al [8] proposed a work for the navigation of the mobile robots in a hybrid sensor network. In this paper we bring up the two novel navigation algorithms for the outdoor environments where the robots are been permitted to travel from one static node to another along a planned path in the sensor field. By the use of this method the robot navigation is been planned without the help of a map. The advantage of this algorithm is being cost effective. A path planning algorithm is also been added to schedule the mobile robots travelling paths which then finally focuses on the shortest distance for robots. Mianxiong Dong et.al [6] has proposed his idea on the multicloud-based system. The cloud concept is been used in the evacuation system to quickly identify the clients location and suggests the evacuation route which gives an immediate response to the client during emergency situations within a short period of time. This cloud-based system technique requires the location of all the people simultaneously and gives various evacuation routes. It is also capable of sharing the information about the disaster. In cloud- based evacuation systems the sensors are been monitored [3] with the use of service instances which provides some basic services. On the occurrence of the disaster many service instances are launched by the evacuation system to meet the potential demand of the evacuation services for the large number of users. So these cloud based evacuation systems maintain active instances in normal time This multicloud-based evacuation (MCES) system is reliable and manageable due to its low cost compared to the single cloud system. Concurrent cloud systems can be launched by MCES during emergency. This paper brings forth three key points. The first idea is to consider the instance of emergency situation in a cloud rather than considering the capacity of the cloud. Next he portrays about the concurrent tackling of emergency in a cloud with quick response. Finally the idea of good maintenance is put forth to prevent the high cost expenses due to instances.

\section{Proposed System Architecture}

The proposed architecture diagram is given in the figure 1 . It has the following phases: (i) Admin Process, (ii) Network Formation, (iii) Destination Navigation and (iv) Emergency Navigation.

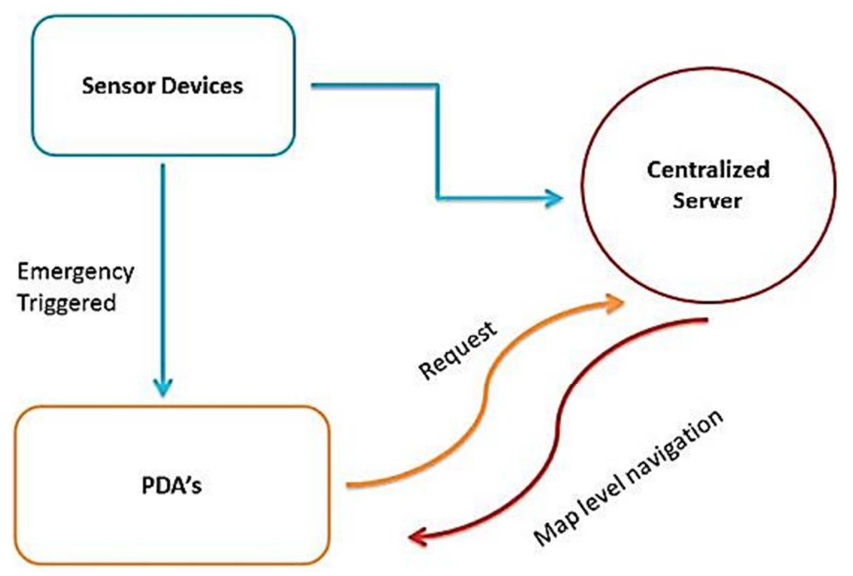

Figure 1. A sample line graph using colors.

\subsection{Admin Process}

The admin should have the prior knowledge about the environment. The admin will pre-process the whole environment for the complete navigation for the users by adding the block details (Peter England, theater, etc...) and 
the exit, the brief description about the block and exit (figure 1). And admin navigate the user by pre-processing the path for source to the destination that the user request.

\subsection{Network Formation}

In Network formation we construct the whole environment, where the environment actors are users, sensors, and the centralized server. Where the sensors are scattered among the environment that sense the environment condition. And the users are with their handheld device that gets connected by the any of the sensor in the environment based on the coverage of the sensor.

\subsection{Destination Navigation}

If the user is in need to get the particular path from the source to the destination. The user request for the path with the destination that user should reach. The centralized server checks with the user's source and destination and find the path for the respective travel. And navigate the user in the map level.

\subsection{Emergency Navigation}

The sensors sense the environmental conditions continuously, if the sensor sense the abnormal values the sensor intimates to the users that connected with the sensor and intimates with the nearby sensors. And the all sensor does the same. And the emergency passes to the whole environment. And the user handheld device gets the navigation from the server that exit as the destination. And the map level navigation has been given to the user's handheld devices.

\section{Constraints in Implementation}

The admin should have the prior knowledge about the environment. The admin will preprocess the whole environment for the complete navigation for the users by adding the block details (Peter England, theater, etc...) and the exit, the brief description about the block and exit. And admin navigate the user by pre-processing the path for source to the destination that the user request. In Network formation we construct the whole environment, where the environment actors are users, sensors, and the centralized server. Where the sensors are scattered among the environment that sense the environment condition. And the users are with their handheld device that gets connected by the any of the sensor in the environment based on the coverage of the sensor. If the user is in need to get the particular path from the source to the destination. The user request for the path with the destination that user should reach. The centralized server checks with the user's source and destination and find the path for the respective travel. And navigate the user in the map level.

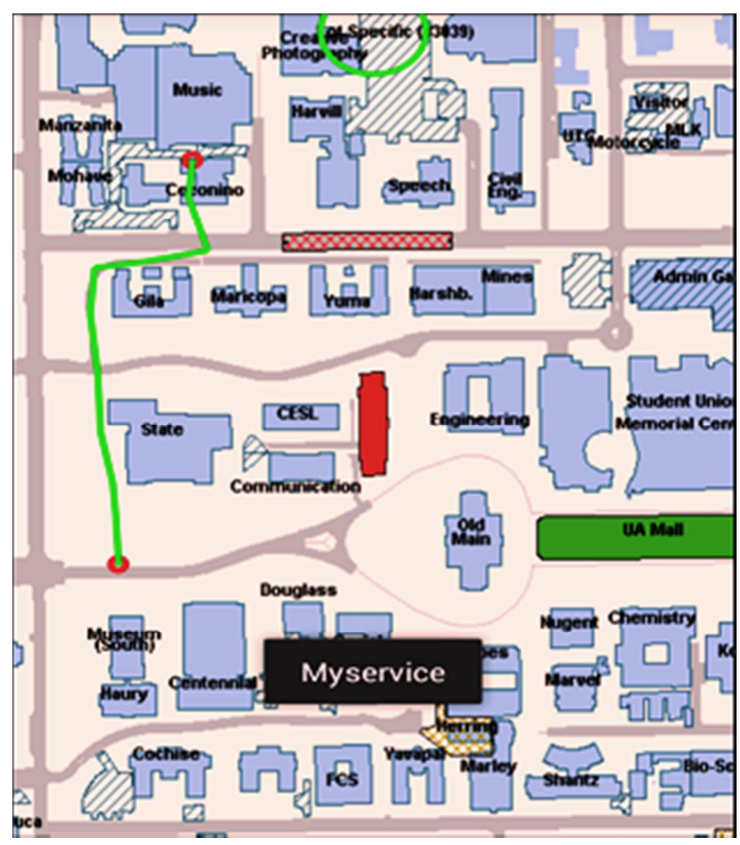

Figure 2. Example of an image with acceptable resolution.

The sensors sense the environmental conditions continuously, if the sensor senses the abnormal values the sensor intimates to the users that connected with the sensor and intimates with the nearby sensors. Whenever new sensors are added the system is subjected to testing [10]. And the all sensor does the same. And the emergency passes to the whole environment. And the user handheld device gets the navigation from the server that exit as the destination (figure 2) and the map level navigation has been given to the user's handheld devices.

\section{Conclusion}

To help individuals in getting away from a risky (perilous range) locale immediately when a crisis happens with ensured wellbeing, while evading extreme clogs and pointless temporary routes has been executed utilizing the earth delineate. The future enhancement is to provide Dynamic Short path, Map level implementation for navigation (from one place to another place) path and Datasets are highly dynamic.

\section{References}

[1] Chen Whang, Hongbo Jiang (2015). "SURF: A Connectivitybased Space Filling Curva Construction Algorithum in High Genus 3D Surface WSNs" IEEE Computer Communication Conference (INFOCOM).

[2] Z Li, J Zhang, X Shen (2016). "Prediction based indoor fire escaping routing with wireless sensor network", peer-to-peer networking and application.

[3] Avinaash Ram S. P., Albert Mayan. J, (2015). "Mobile Attendance Management and Employee Registration", ARPN Journal of Engineering and Applied Sciences, Vol: 10, Issue 8, pp: 3727-3730, ISSN: 1819-6608. 
[4] WMYM Bejuri, MM Mohamad, R Zahilah, Raja Mohd Radzi, (2015). "Emergency rescue localization using GPS, wireless LAN and camera", International journal of software engineering, vol. 9. no 9.

[5] Lin Wang, Yuan He, Wenyuan Liu, Nan Jing, Jiliang Wang, Yunhao Liu, (2015). "On oscillation free emergency navigation via wireless sensor networks", IEEE transactions vol. 14, Issue: 10.

[6] Y. Song, B. Wang, Z. Shi, K. Pattipati, and S. Gupta, (2014). "Distributed algorithms for energy-efficient even selfdeployment in mobile sensor networks," IEEE Trans. Mobile Compute, vol. 13, no. 5, pp. 1035-1047.

[7] Mianxiong Dong, he Li, Kaoru Ota, Laurence T. Yang, Haojin Zhu, (2014). "Multicloud- Based Evacuation Services for Emergency Management:", IEEE Computer Society.
[8] Jiliang Wang, (2013). "Sensor Network Navigation without Location", IEEE.

[9] Yovan Felix. A, Pamila. A (2016). "Joint threshold administration model in relational databases to enhances the security", International Journal of Control Theory and Applications, Vol 9(12), pp. 5765-5769.

[10] Albert Mayan. J, Dr. T. Ravi, (2015). "Structural Software Testing: Hybrid Algorithm For Optimal Test Sequence Selection During Regression Testing", International Journal of Engineering and Technology, Vol: 7, Issue:1, pp: 270-279, ISSN: 0975-4024.

[11] Zheng Zhang, Zhenbo Li, Dawei Zhang, Jiapin Chen,(2013). "Path Planning and Navigation for Mobile Robots in a Hybrid Sensor Network without Prior Location Information", SAGE journals. 